\title{
Future physics with polarized ep colliders
}

\author{
A. De Roeck ${ }^{\mathrm{a} *}$ \\ ${ }^{\mathrm{a}}$ CERN, 1211 Geneva 23, Switzerland
}

We discuss some of the physics opportunities at polarized ep-colliders. Recent studies for polarized ep-colliders include HERA, EIC and THERA.

\section{Introduction}

The commissioning of the first electron-proton collider HERA (27.5 GeV electrons on $820 \mathrm{GeV}$ protons) eight years ago opened up a completely new kinematical domain in deep inelastic scattering (DIS), and the two HERA experiments have provided a multitude of new insights into the structure of the proton and the photon since then. It is therefore natural to assume that the operation of $e p$-colliders with polarized proton and electron beams will add vital new information to our picture of the spin structure of the nucleon. Possible high energy ep-collider projects presently under discussion are listed in Table 1 .

HERA has just been upgraded to reach a higher luminosity. It has excellent detectors, and the electron beam is already polarized. The polarization of the proton beam is technically more involved than for the electron beam, since protons do not polarize naturally in the HERA ring. Hence beams from a source of polarized protons have to be accelerated through the whole chain and the polarization has to be kept during the process. The technical aspects of this project are elaborated in [1]. Based on these studies, it seems realistic to assume that HERA could be operated with polarized electron and proton beams, each polarized to about $70 \%$, acumulating a luminosity of $500 \mathrm{pb}^{-1}$ when integrated over several years.

The Electron Ion Collider (EIC) [2] -if built at BNL- will need a polarized electron accelerator, most likely a LINAC, added to the RHIC po-

\footnotetext{
*Invited talk presented at the "International Workshop on the Spin Structure of the Proton and Polarized Collider Physics", ECT*, Trento, Italy, July 23-28, 2001.
}

larized proton rings, and will probably also need a dedicated experiment. THERA [3] will need TESLA to be built at DESY (or tangential to the TEVATRON), polarized protons in the proton ring, and a new detector. The advantage of the EIC is its large reachable luminosity, imperative for polarized studies. At HERA the luminosity is (just) enough for most topics but its advantage lies in its larger kinematical reach. THERA reaches even further in the $x$ and $Q^{2}$ plane, but its relatively low luminosity of $40 \mathrm{pb}^{-1} /$ year may be a handicap for many studies.

Most detailed studies for polarized $e p$ scattering at a collider have been made in a series of workshops for HERA [4, 5] and these will serve as a basis for the discussion in this paper. Many of these studies have been carried over to EIC in the last two years. For THERA polarized studies have only been briefly explored so far.

Here we will discuss measurements of $g_{1}\left(x, Q^{2}\right)$ at low $x$, the extraction of the polarized gluon density $\Delta G\left(x, Q^{2}\right)$ in a wide kinematic range using several processes, the extraction of the spin structure function $g_{5}\left(x, Q^{2}\right)$ from charged current events, photoproduction studies and studies on the helicity structure of the high $Q^{2}$ region.

\section{The polarized structure function $g_{1}\left(x, Q^{2}\right)$}

The outstanding advantage of a high energy epcollider is that it can measure structure functions at very small $x$ and very large $Q^{2}$. The kinematical reach is shown in Fig. 1 for the different machines. The region covered by present fixed target experiments is shown as well. The region 
Table 1

Possible future $e p$-collider facilities for polarized scattering

\begin{tabular}{|l|l|l|}
\hline Machine & Lumi/year & $\sqrt{s}$ \\
\hline HERA & $150 \mathrm{pb}^{-1}$ & $320 \mathrm{GeV}$ \\
$\begin{array}{l}\text { Electron-Ion } \\
\text { Collider (EIC) }\end{array}$ & $4 \mathrm{fb}^{-1}$ & $30-100 \mathrm{GeV}$ \\
$\begin{array}{l}\text { THERA } \\
\text { (TESLA } \otimes \text { HERA) }\end{array}$ & $40(250) \mathrm{pb}^{-1}$ & $1-1.6 \mathrm{TeV}$ \\
\hline
\end{tabular}

can be extended by several orders of magnitude both in $x$ and $Q^{2}$. The regions of the three colliders overlap. At HERA measurements at values of $Q^{2}$ up to $2 \cdot 10^{4} \mathrm{GeV}^{2}$ are reached, and values of $x$ below $10^{-5}$. Hence such data will allow for detailed QCD tests similar to the ones in the unpolarized case.

An example for HERA is shown in Fig. 2. The low- $x$ behaviour of $g_{1}$ is indicated in the figure: the straight line is an extrapolation based on Regge phenomenology, and the upper curve presents a scenario suggested in [6] where $g_{1}$ rises as $1 /\left(x \ln ^{2}(x)\right)$, which is the maximally singular behaviour still consistent with integrability requirements. The low $x$ behaviour of $g_{1}$ by itself is an interesting topic as discussed in [7].

An EIC has as expected a more restricted range, as shown in Fig. 3. However the error bars can be substantially smaller due to the high luminosity of $4 \mathrm{fb}^{-1} /$ year. THERA would reach $x$ values down to $10^{-6}$, but needs at least one $\mathrm{fb}^{-1}$ of data.

Note that the expected asymmetries at low $x$, i.e. $x \sim 10^{-4}$ are relatively small, about $10^{-3}$, which puts strong requirements on the control of the systematic effects, as discussed in [5]. At the lowest $x$ values at THERA, the asymmetries may be even smaller, and the potential to actually extract a polarized structure function at these values still needs to demonstrated.

It would be very advantageous to have polarized low- $x$ neutron data which would enable to measure singlet and non-singlet polarized structure functions at low $x$. A study was made using polarized ${ }^{3} \mathrm{He}$ at HERA [6], from which $g_{1}^{n}$ can be extracted. If the machine can provide polar-

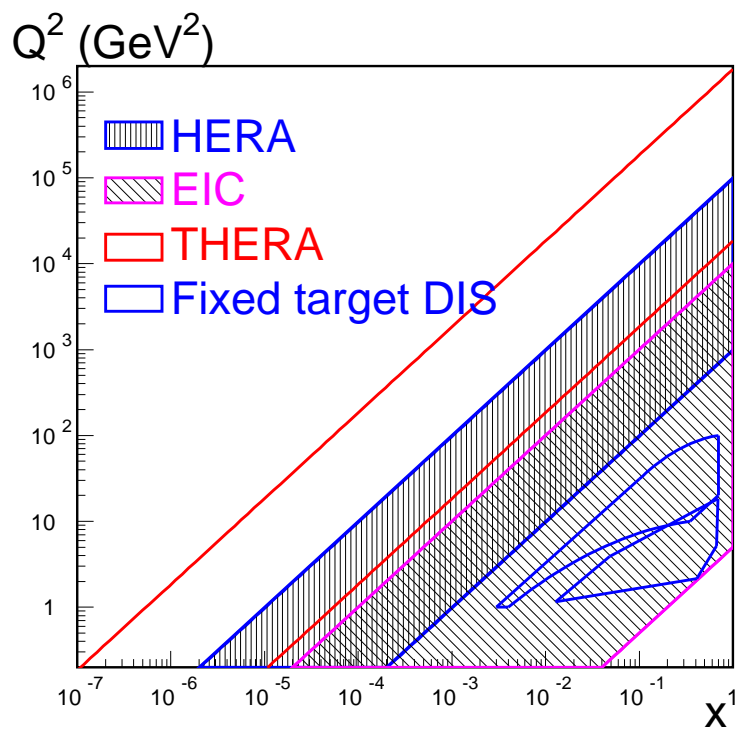

Figure 1. Measurable $x-Q^{2}$ region for a polarized HERA with the presently explored regions by fixed targer experiments. The energies used are for THERA: $E_{p}=920, E_{e}=500$ GeV; HERA: $E_{p}=920, E_{e}=27 \mathrm{GeV}$; eRHIC: $E_{p}=250, E_{e}=10 \mathrm{GeV}$ and $E_{p}=50, E_{e}=5$ $\mathrm{GeV}$, and roughly $0.01<y<1.0$.

ized ${ }^{3} \mathrm{He}$ with a luminosity comparable to the one for the protons, such a program can be carried out. A recent new idea is to store polarized deutrons [8]. Due to the small gyromagnetic anomaly value the storage and acceleration problems are less severe for deutrons and it could be possible to keep the polarization at HERA even without the use of Siberian snake magnets for deuterons. However the spin cannot be flipped by spin rotators from transverse to longitudinal polarization in the interaction regions, and other means, such as the recently suggested use of external radial frequency fields must be considered for arranging that the spins align longitudinally at the interaction points. For polarized deuteron beams the changes to the present HERA machine could be more modest, and, if one can instrument the region around the beam-pipe to tag the spectator in 


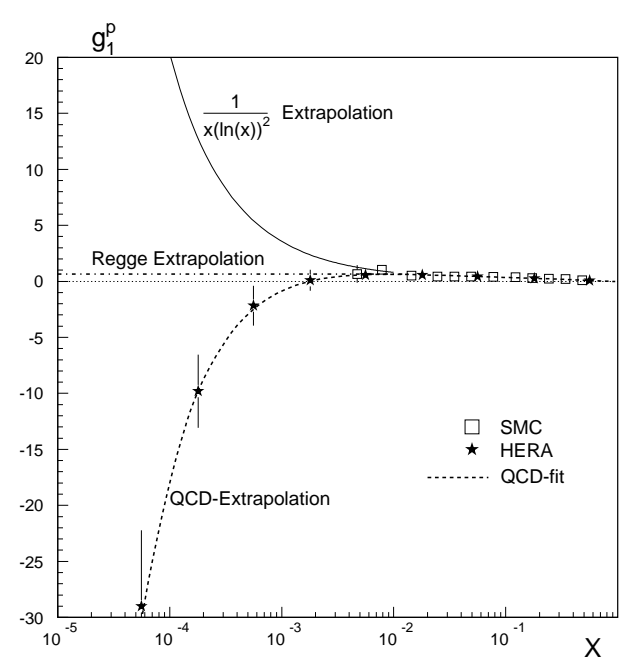

Figure 2. The statistical uncertainty on the structure function $g_{1}$ of the proton measurable at HERA, evolved to a value of $Q^{2}=10 \mathrm{GeV}^{2}$ for an integrated luminosity of $500 \mathrm{pb}^{-1}$. The SMC measurements are shown for comparison.

the deuteron nucleus, it can give simultaneously samples of scattering on $p$ and $n$. An intense polarized deuteron source is however needed. Furthermore, so far the accelerating and storing of polarized deuteron beams has been studied to a much lesser extend than for polarized protons.

With proton and neutron data available one can measure the Bjorken sum rule $\int g_{1}^{p} d x-$ $\int g_{1}^{n} d x$, presently measured to about $10 \%$ precision. E.g. EIC data will allow to measure this sum rule, a key test for $\mathrm{QCD}$, to a few $\%$ precision.

\section{The polarized gluon distribution $\Delta G\left(x, Q^{2}\right)$}

The high quality $g_{1}$ data from the fixed target experiments allows for quantitative QCD studies of the polarized structure function data, from which polarized parton distributions are extracted [6].

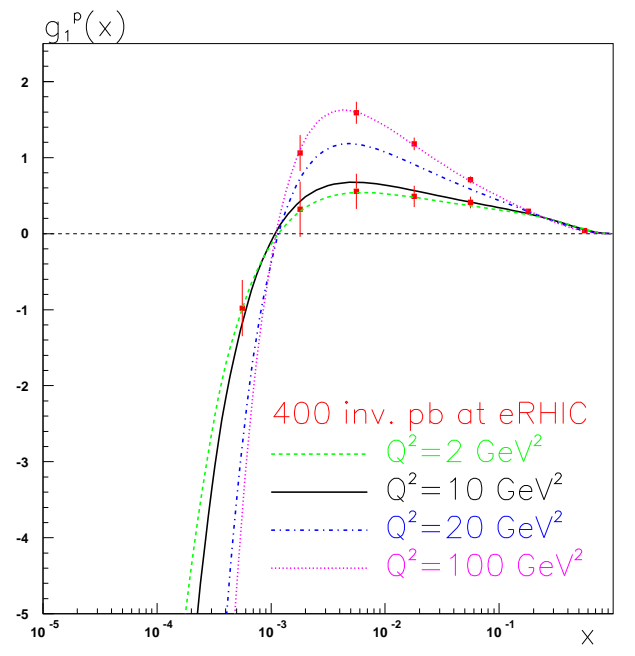

Figure 3. The statistical uncertainty on the structure function $g_{1}$ of the proton measurable at $\mathrm{EIC}$, for an integrated luminosity of $400 \mathrm{pb}^{-1}$.

The relatively small contribution of the quarks to the spin of the proton, as follows from the 'spin puzzle', leads to a rather large value of the polarized gluon. The precision is however rather limited. The measurements from present day data for the first moment of the polarized gluon distribution give typically $\int \Delta G(x) \mathrm{d} x=$ $0.9 \pm 0.3(\exp ) \pm 1.0$ (theory) at $Q^{2}=1 \mathrm{GeV}^{2}$. The theoretical error on this quantity is dominated by the extrapolation into the yet unmeasured low- $x$ region. Including future HERA data will improve the experimental error to about 0.2 . The theoretical error is expected to decrease by more than a factor 2 once $g_{1}\left(x, Q^{2}\right)$ is measured at low $x$. At an EIC one expects to reduce the statistical uncertainty even to 0.08 with about $10 \mathrm{fb}^{-1}$, as discussed in [9].

It is crucial for our full understanding of the proton spin that the prediction of a large polarized gluon is confirmed by direct measurements of $\Delta G$. HERA has shown that the large centre of mass system (CMS) energy allows for several processes to be used to extract the unpolarized 

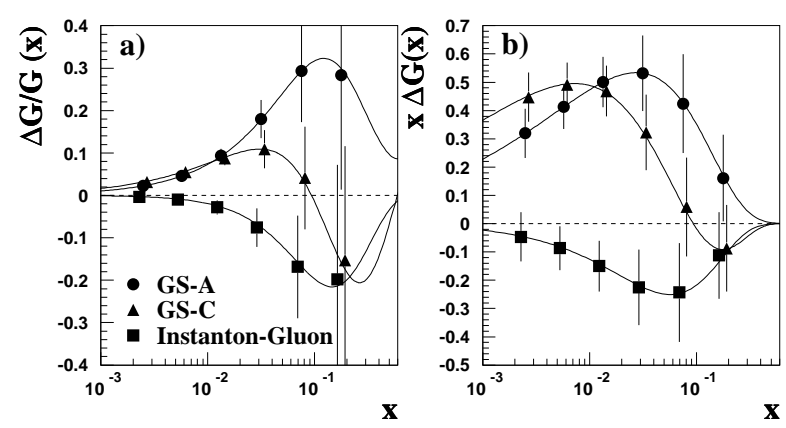

Figure 4. High $p_{t}$ hadrons: Sensitivity to $\Delta G / G$ (a) and $x \Delta G$ (b) for three different polarized gluon distributions shown as solid lines and a luminosity of $500 \mathrm{pb}^{-1}$, for $Q^{2}=20 \mathrm{GeV}^{2}$.

gluon distribution. These include jet and high $p_{t}$ hadron production, charm production both in DIS and photoproduction, and correlations between multiplicities of the current and target hemisphere of the events in the Breit frame.

The most promising process for a direct extraction of $\Delta G$ at HERA is di-jet production, as discussed in 10]. The underlying idea is to isolate boson-gluon fusion events, i.e. a process where the gluon distribution enters at the Born level. Jets are selected with a $p_{t}>5 \mathrm{GeV}$ and are restricted to the acceptance of a typical existing HERA detector by the requirement $\left|\eta_{L A B}^{j e t}\right|<2.8$, where $\eta_{L A B}^{j e t}$ is the pseudo-rapidity in the laboratory system. The resulting measurable range in $x$ (of the gluon) is $0.002<x<0.2$ with typically 6 measurable data points. At an EIC the measurable range is reduced to $0.02<x<0.3$. The reach at THERA is $0.0005<x<0.1$ but an event sample of several hundered $\mathrm{pb}^{-1}$ will be needed.

Instead of jets, single hadrons with high transverse momentum $p_{t}$ opposite in azimuthal angle in the $\gamma^{*} p$ frame can be used. [11]. In a study for HERA two charged tracks with a $p_{t}$ larger than $1.5 \mathrm{GeV}$ are required [12]. The resulting gluon distribution is shown in Fig. 1 and compared to several predicted gluon distributions. A
Table 2

The expected statistical uncertainty in the determination of the first moment of the gluon distribution at $Q^{2}=1 \mathrm{GeV}^{2}\left(500 \mathrm{pb}^{-1}\right)$, see text.

\begin{tabular}{||l|c||}
\hline \hline Analysis Type & $\boldsymbol{\delta}\left(\int \boldsymbol{\Delta} \boldsymbol{G} \mathbf{d} \boldsymbol{x}\right)$ \\
\hline \hline $1 . g_{1}$ fixed target & 0.3 \\
\hline 2. $g_{1}$ fixed target + HERA & 0.2 \\
\hline 3. di-jets at HERA & 0.2 \\
\hline 4. combined $2 \& 3$ & 0.1 \\
\hline \hline
\end{tabular}

similar level of discriminating power as for the di-jet events is obtained, except in the highest $x$ region, where the di-jets are superior. These measurements allow for the determination of the shape of $\Delta G(x)$. The errors on the individual points for the di-jet measurement on $\Delta G(x) / G(x)$ are in the range from 0.007 to 0.1 . The total error on $\Delta G(x) / G(x)$ in the complete range is 0.02 .

An exploratory study was made, using the values of $\Delta G(x)$ obtained from the di-jet analysis as an extra constraint in the fit of $g_{1}$ data discussed above. The improvement of the errors on the first moment of $\Delta G$ due to the inclusion of di-jet data is shown in Table 2. The first two rows give the values quoted before, namely for the NLO QCD analysis without and with projected HERA data for $g_{1}$. The third row shows the expected error if only the di-jet asymmetry is added to the fixed target $g_{1}$ data, and the fourth row shows the total improvement using all available information.

\section{Polarized quark distributions}

In present fixed target experiments information on the flavour decomposition can be obtained from semi-inclusive measurements, i.e. measurements where a final state hadron is tagged. At HERA one has the additional option to study quark flavours in a more inclusive, and therefore fragmentation function independent mode, via charged current interactions. The asymmetry is defined by

$A^{W \mp}=\frac{d \sigma_{\uparrow \downarrow}^{W^{\mp}}-d \sigma_{\uparrow \uparrow}^{W^{\mp}}}{d \sigma_{\uparrow \downarrow}^{W^{\mp}}+d \sigma_{\uparrow \uparrow}^{W \mp}} \quad \approx \frac{g_{5}^{W^{\mp}}}{F_{1}^{W \mp}}$ 


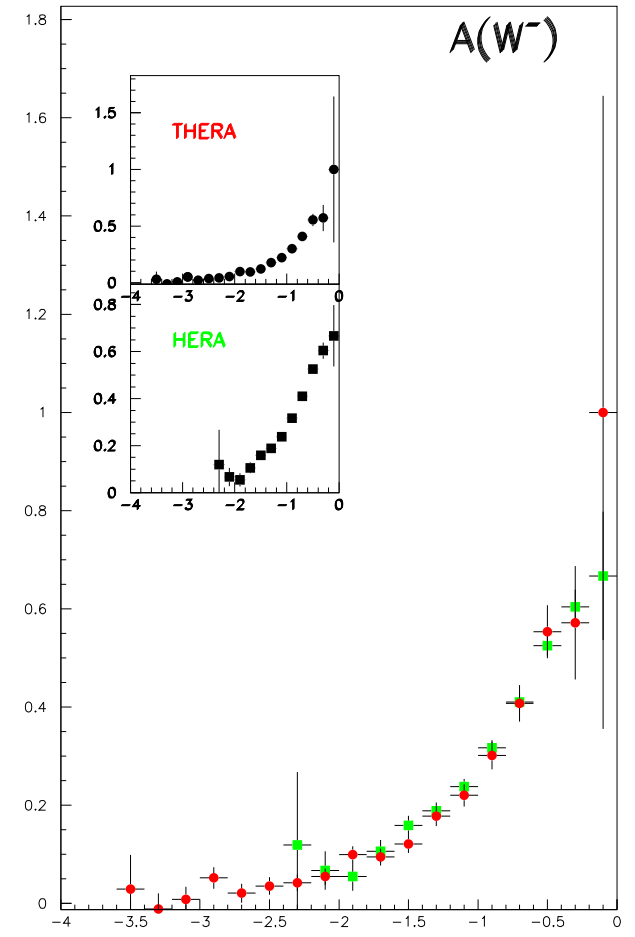

Figure 5. Spin asymmetries $A^{W^{-}}$for charged current events for a total luminosity of $500 \mathrm{pb}^{-1}$. The error bars represent the statistical uncertainty of the measurement.

with $g_{5}^{W^{-}}=\Delta u+\Delta c-\Delta \bar{d}-\Delta \bar{s}, g_{5}^{W^{+}}=$ $\Delta d+\Delta s-\Delta \bar{u}-\Delta \bar{c}$. A Monte Carlo study, including detector effects, was made for the measurements of the asymmetry and the extraction of $g_{5}$ [13]. The total missing transverse momentum (which is a signal for the escaping neutrino) was required to be $P_{\text {Tmiss }}>15 \mathrm{GeV}$, and the region $Q^{2}>225 \mathrm{GeV}^{2}$ has been selected for this analysis. The results for the asymmetries for both HERA and THERA are shown in Fig. 每. The error bars indicate the statistical precision of the measurement. The asymmetries are very large and significant measurements can be produced at THERA down to $x=10^{-3}$. These charged current measurements, with both $e^{+}$and $e^{-}$beams, can be used to extract e.g. the $\Delta u$ and $\Delta d$ distributions.

\section{Photoproduction}

Photoproduction processes have been shown to be sensitive probes for the polarized parton structure in the proton AND in the photon. Single jet production has been studied in [4]. In [14] it was shown that also a high $p_{t}$ track analyses yields a similar sensitivity, with only modest luminosity requirements.

Polarized photoproduction of di-jets has been investigated in detail, including in particular effects due to parton showering, hadronization, jet finding and jet clustering. It could be demonstrated that, although these effects yield sizable corrections, the measurable asymmetry will largely be preserved at the hadron level [14]. An example for the correspondence of parton and hadron level asymmetries is shown in Fig. 6, obtained with a moderate integrated luminosity of only $50 \mathrm{pb}^{-1}$. A first idea on the discriminative power on the photon structure of future measurements can however be gained by comparing the predictions obtained with the two (minimal and maximal) polarization scenarios proposed in [15], as done in Fig. 6 .

Finally, it can be pointed out that a measurement of the total polarized photoproduction cross section $\Delta \sigma_{\gamma p}(\nu)$ as function of the photonproton CMS energy $\nu$ at HERA would contribute to a precise understanding of the Drell-HearnGerasimov sum rule, as discussed in 16. In particular the Regge behaviour of the energy dependence of the polarized cross section can be tested. The sensitivity which could be reached with EIC is about one order of magnitude larger than the one for HERA.

\section{Effects at high $Q^{2}$}

At an $e p$-collider the region of high $Q^{2}$ offers the largest chance of discovering new physics. Such new physics could manifest itself through the production of new particles, such as Leptoquarks or SUSY particles in RP violating models, contact interactions, etc. A general study was made 


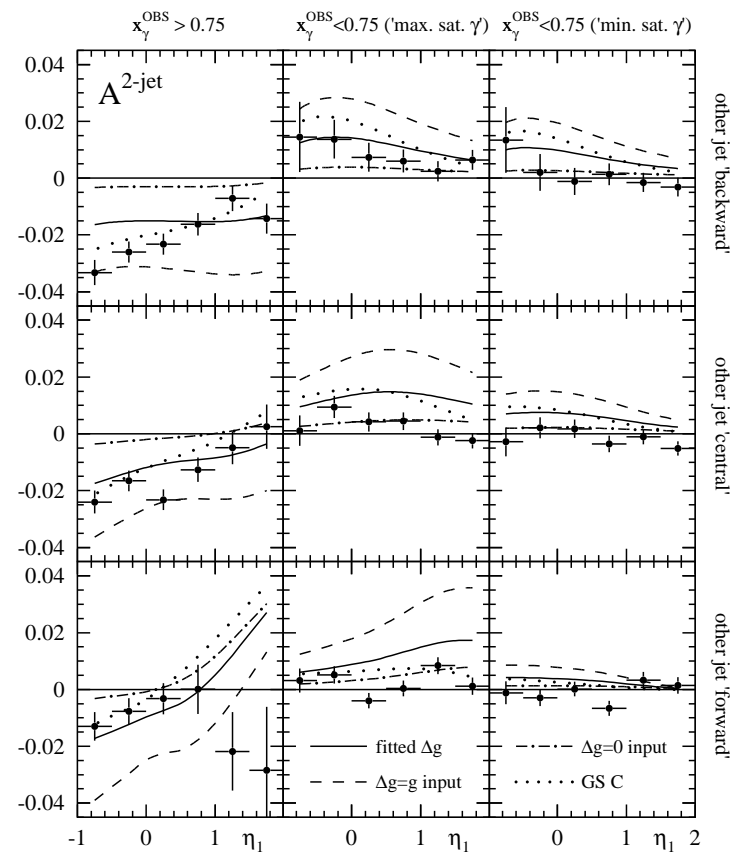

Figure 6. Polarized photoproduction of di-jets $\left(E_{T, 1}>8 \mathrm{GeV}, E_{T, 2}>6 \mathrm{GeV}\right)$ : asymmetries for direct (first column) and resolved (second and third column) photon contributions as function of the rapidity of the first jet and for different orientations of the second jet. Second and third column correspond to different scenarios for the parton content of the polarized photon suggested in 15. The error bars shown correspond to a Monte Carlo sample of $50 \mathrm{pb}^{-1}$.

based on the contact interaction formalism [17. It was demonstrated that a fully polarized HERA would be very instrumental in disentangling the chiral structure of the new interactions. With $250 \mathrm{pb}^{-1}$ data samples for polarized $e^{+}$and $e^{-}$ beams, for each of the 2 spin orientations, the asymmetries are sensitive to contact interactions to scales larger than $7 \mathrm{TeV}$ (95\% C.L.). In the presence of a signal these different combinations of cross sections into the seven different asymmetries allow a complete identification of the chiral structure of the new interactions, i.e. whether the interactions are LL, RR, LR or LR or a combination of those (where $\mathrm{L}$ and $\mathrm{R}$ denote the left and right handed fermion helicities for the lepton and quark respectively).

This study has been further extended to the special case of leptoquark-like production [18]: asymmetries like the ones above would allow to pin down the chiral properties of the couplings to these new particles.

An interesting possibility is the effect induced by QCD instantons [19] to the proton structure function. Non-perturbative instanton fluctuations describe the quantum tunnelling between different gauge rotated classical vacua in QCD. Due to the quark helicity flip at the quarkinstanton vertex, the contribution to the spindependent cross sections of instantons is very different from the one of the perturbative quarkgluon vertex. Furthermore, in the instanton liquid model [20] the contribution of instantons to the proton structure is expected to become increasingly more important with increasing $Q^{2}$ 19].

These high $Q^{2}$ studies may be most relevant for HERA and THERA. For THERA both $e^{+}$and $e^{-}$ beams will be needed and minimum integrated luminosities of order $100-200 \mathrm{pb}^{-1} /$ beam.

\section{Other Topics}

Many other topics on polarization can be studied at an $e p$-collider, e.g. target fragmentation properties, transversity, DHG, issues related to diffraction, spin transfer in $\Lambda$ polarization, semiinclusive measurements to extract the polarized quark distributions, etc. Further details can be found in [5].

\section{Conclusions}

Polarized $e p$-colliders with a centre of mass energies of $100-1000 \mathrm{GeV}$ will allow to make unique measurements in polarized deep inelastic scattering as well as photoproduction.

The necessity for low- $x$ measurements of the 
structure functions, and determination of the polarized gluon distribution $\Delta G$ have been wildly advocated by the spin physics community over the last five years. ep-colliders can play a pivotal role in this field since they are able to give conclusive insight on both of these issues.

Polarized ep-colliders will also contribute to the flavour decomposition of the quark spin distributions, and the very intriguing possibility to measure polarized parton distributions in the photon. Furthermore -not discussed in this review- new insight is expected on spin transfer in quark fragmentation and spin effects in diffractive scattering. Finally, a polarized HERA or THERA will be very instrumental in the study and interpretation of possible deviations from the Standard Model expectation in the high- $Q^{2}$ region, if observed.

However the required luminosity for any such machine should be at least $100 \mathrm{pb}^{-1} /$ year, preferably even more. The polarizations of the beams should be larger than $50-60 \%$. If EIC will be the world's only polarized collider, it would be useful to consider having an extention of the CMS energy, via an upgrade of the $e$-LINAC energy e.g. from 10 to $20-30 \mathrm{GeV}$, to cover an as large kinematic region as possible. If new detectors are required or can be afforded, particular care should be taken on the design, learning from shortcomings from the present HERA detectors, in e.g. the small angle instrumentation [21]. Polarized deuterons seem an attractive addition or even alternative to polarized protons, and are perhaps easier to accelerate and store, but detailed machine studies need to be performed to confirm these ideas.

In all, polarized scattering experiments have in the last decades often revealed surprises, hence a polarized collider seems an attractive future option with a rich program.

\section{REFERENCES}

1. SPIN at HERA Collaboration, L.V. Alekseeva et al., University of Michigan preprint UM-HE-96-20 (1996); UM-HE-99-05 (1999); http://www-mpy.desy.de/proton_pol/.

2. see http://quark.phy.bnl.gov/ raju/yale_
eRHIC.html

3. Appendix to the TESLA TDR: THERA: electron proton scattering at $\sqrt{s} \sim 1 \mathrm{TeV}$, H. Abramowicz et al., DESY-01-011 (2001).

4. Proceedings of the workshop 'Physics with Polarized Protons at HERA', Eds. A. De Roeck and T. Gehrmann DESY-98-01.

5. Proceedings of the workshop "Polarized Protons at High Energies - Accelerator Challenges and Physics Opportunities" ed. A. De Roeck, D. Barber and G. Rädel, DESYPROC-1999-03 (1999).

6. A. De Roeck et al., Eur. Phys. J. C6 (1999) 131

7. J. Kwiecinski, Acta Phys. Polon. B29 (1998) 1201.

8. Y. Derbenev, private communications, and V. Anferov and Y. Derbenev, Phys. Rev. PRST-AB 12, 1345 (2000).

A. Skrinsky, proc. of the workshop EPIC99, ed. L.C. Bland et al., Bloomington, USA, April 1999, World Scientific.

9. J. Lichtenstadt, these proceedings.

10. G. Radel, these proceedings.

11. A. Bravar, D. von Harrach and A. Kotzinian, Phys. Lett. B421 (1998) 359.

12. G. Rädel and A. De Roeck, (hep-ph/9711382).

13. J.G. Contreras, A. De Roeck and M. Maul, (hep-ph/9711418).

14. J. Butterworth, N. Goodman, M. Stratmann and W. Vogelsang, (hep-ph/9711250).

15. M. Glück and W. Vogelsang, Z. Phys. C55 (1992) 353; C57 (1993) 309;

M. Glück, M. Stratmann and W. Vogelsang, Phys. Lett. B337 (1994) 373.

16. S.D. Bass, and A. De Roeck, Eur. Phys. J. C18 (2001) 538.

17. J.M. Virey, (hep-ph/9710423).

18. P. Taxil, E. Tugcu, J.M. Virey, Eur. Phys. J. C14 (2000) 178.

19. N. Kochelev, Phys. Rev. D57 (1998) 5542.

20. T. Schäfer and E.V. Shuryak, Rev. Mod. Phys. 70 (1998) 426

D.I. Diakonov, hep-ph/9602375).

21. W. Krasny, these proceedings. 